\title{
The Clinical Efficacy and Safety of Autologous Activated Platelet-Rich Plasma Injection in Androgenetic Alopecia
}

\author{
Niluka Dilrukshi Paththinige ${ }^{a} \quad J a n a k a$ K.W. Akarawita ${ }^{a}$ Geetha Jeganathan ${ }^{b}$ \\ a Department of Dermatology, National Hospital, Colombo, Sri Lanka; ${ }^{b}$ Department of Transfusion Medicine, \\ National Hospital, Colombo, Sri Lanka
}

\section{Keywords}

Platelet-rich plasma - Androgenetic alopecia - Efficacy . Safety

\begin{abstract}
Introduction: Androgenetic alopecia (AGA) is a common, chronic hair loss disorder. Platelet-rich plasma (PRP) is a novel therapeutic tool for AGA. The objective of this study was to assess the clinical efficacy and safety of autologous activated PRP injections in AGA. Methods: Twenty-four AGA patients were enrolled in the study. All the patients received 4 PRP treatments at baseline (T1), 3 weeks (T2), 6 weeks (T3), and at 14 weeks (T5). We evaluated hair density (hairs $/ \mathrm{cm}^{2}$ ) at 6 time points (T1-T3, T4 [9 weeks], T5, and T6 [7 months]) and patient satisfaction was assessed at T6 with a patient satisfaction questionnaire. Hair count (hairs $/ 0.48 \mathrm{~cm}^{2}$ ) was assessed using dermoscopic photographs and hair density (hairs $/ \mathrm{cm}^{2}$ ) was calculated accordingly. Results: Twentythree men and one woman were included with baseline hair density of $102.25 \pm 18.463$. Hair density significantly increased at all time points with $p<0.001$ compared to the baseline. Patients were satisfied with a mean result rating of 72.92 on a linear analogue scale of $0-100$. Other than mild pain felt during injections, no remarkable adverse effects were noted. Conclusions: PRP injections may have a positive therapeutic effect on AGA without major side effects.
\end{abstract}

(c) 2019 S. Karger AG, Basel

\section{KARGER}

() 2019 S. Karger AG, Basel

E-Mail karger@karger.com

www.karger.com/sad

\section{Introduction}

Androgenetic alopecia (AGA) is a common, chronic hair loss disorder, which is due to a combination of genetic predisposition and the effect of androgens on scalp hair follicles. The prevalence of AGA increases with advancing age; however, the age of onset and rate of progression can be varying [1]. The morbidity of AGA is predominantly psychological. Since hair is an essential part of an individual's self-image, significant hair loss can have direct effects on personal attractiveness. Irrespective of age and gender, a person's quality of life may be impaired significantly with the diagnosis of AGA $[2,3]$. Current treatment options for AGA are mainly focused on promotion of hair follicle cell proliferation as well as differentiation during the hair growth cycle. Minoxidil and finasteride are the only Food and Drug Administration (FDA)-approved drug therapies for AGA, and these can effectively arrest hair loss, but hair regrowth with these are limited [4]. Local and systemic adverse effects, low patient compliance, as well as low satisfaction rate of the available therapeutic options has led to the trial of novel treatment strategies for AGA $[1-3,5,6]$.

Definition of platelet-rich plasma (PRP) is a volume of the plasma fraction of blood which has an above baseline platelet concentration [7]. PRP is used to promote hair growth due to its regenerative potential and this relies on

Niluka Dilrukshi Paththinige

Department of Dermatology, National Hospital of Sri Lanka

249/2, Reality homes, 2nd Lane, Pipe Road

Battaramulla, Colombo 10120 (Sri Lanka)

E-Mail nilupaththinige@gmail.com 
the amounts of growth factors released following activation of platelets $[8,9]$. There are several growth factors involved in AGA and they are transforming growth factor, platelet-derived growth factor, vascular endothelial growth factor, insulin-like growth factor, and their isoforms [10-12]. These growth factors usually act in the hair follicular unit stem cells, where they bind to their relevant receptors. Epidermal cells and sebaceous glands are originated from ectodermally originated stem cells in the bulge area of the hair follicle. Mesenchymal originated germinative cells are found at the dermal papilla, in the matrix. Cellular interactions between these cells and binding of growth factors lead to activation of the proliferative phase of the hair, which gives rise to the future follicular unit [13]. Hence, PRP could be considered as a potential treatment modality for AGA.

The objective of this study was the assessment of the clinical efficacy and safety of autologous activated PRP (AA-PRP) injections on AGA patients.

\section{Materials and Methods}

\section{Subjects}

A total of 28 subjects ( 27 males, 1 female, in an age range of 20-50 years) with AGA were enrolled in the study. None of the participants were on any topical or systemic therapy for hair loss for the previous 6 months. Participants with dermatological diseases affecting the scalp, history of immunosuppression (longterm steroid therapy, malignancy, or chemotherapy), keloidal tendency, autoimmune diseases, platelet dysfunction disorders, hematological diseases, and on anticoagulation therapy were also excluded.

Diagnosis of AGA was made based on clinical and trichoscopic features (diversity of hair shaft diameter between affected and normal areas) and by exclusion of other contributory causes of hair loss, such as thyroid dysfunction, anemia, or polycystic ovary syndrome with detailed medical history, clinical examination, and blood tests. Blood tests included:

- Full blood count

- TSH and free T4 levels

- For women - follicle stimulating hormone, luteinizing hormone, and testosterone levels

The extent and staging of AGA were assessed utilizing the Hamilton-Norwood scale for males and Ludwig scale for females. Written informed consent was taken from all the patients before participating in the study.

\section{Treatment Protocol}

PRP was prepared using Dr. PRP kit with approval of the transfusion physician. Initially $18 \mathrm{~mL}$ of blood was collected from antecubital vein of the patient to a 20 -mL syringe with $2 \mathrm{~mL}$ of sodium citrate as an anticoagulant, and final volume of $20 \mathrm{~mL}$ was introduced into PRP kit. First centrifugation (male - 3,000 rpm for 4 min, female $-3,000 \mathrm{rpm}$ for $3 \mathrm{~min}$ ) separated the plasma layer from the red blood cell layer. Then the height of the separated boundary was adjusted to the indicated point by pushing up or pulling down the adjusting knob located at the lower part of the kit. After this, complete blocking of the plasma and the red blood cell layer was done by fastening the adjusting knob and then the valve counterclockwise. Finally, the adjusting knob was fastened counterclockwise again. The fastened PRP kit was directed for second centrifugation (6 min at 3,200 rpm) to enrich the concentrated platelet. After the second centrifugation, the PRP kit was kept in its upright position and platelet-poor plasma was taken out slowly from the upper part leaving $4 \mathrm{~mL}$ at the base. Then gentle shaking was done and the remaining $4 \mathrm{~mL}$ of PRP were taken into a 10-mL syringe. The activation of platelets in PRP was done immediately before the treatment by addition of calcium gluconate in a $1: 9$ ratio $(0.1 \mathrm{~mL}$ calcium gluconate per $0.9 \mathrm{~mL}$ of PRP). AAPRP was loaded in 1-mL syringes before injection.

Patients were advised to wash their hair on the day before the treatment. Application of local anesthetic cream to the treatment area was done $1 \mathrm{~h}$ before injection time. Scalp skin was cleaned with $70 \%$ alcohol before the procedure. PRP $\left(0.1 \mathrm{~mL} / \mathrm{cm}^{2}\right)$ was injected into the affected areas using $1-\mathrm{mL}$ syringes with a $25-\mathrm{G}$ needle. Nappage technique (injections $1 \mathrm{~cm}$ apart, in a linear manner) was performed in a depth of $1.5-2.5 \mathrm{~mm}$ under aseptic precautions.

Our protocol proposed four treatment sessions with initial three treatments in an interval of 3 weeks and a booster session performed at 14 weeks from the baseline treatment ( 2 months after the 3rd treatment session).

\section{Assessment Criteria}

Evaluation was done at six time points $(\mathrm{T})$ for all the participants: T1, at baseline; T2, at 3 weeks; T3, at 6 weeks; T4, at 9 weeks; T5, at 14 weeks; and T6, 7 months from baseline. We evaluated hair density (hairs $/ \mathrm{cm}^{2}$ ) and any adverse effects at each time point, and patient's satisfaction was assessed at T6. Evaluation methods included macrophotography and dermoscopic photography. In one individual patient, the same area was focused for dermoscopic photographs at each evaluation. The hair count (hairs $/ 0.48 \mathrm{~cm}^{2}$ ) was assessed manually by the chief evaluator using dermoscopic photographs and hair density (hairs $/ \mathrm{cm}^{2}$ ) was calculated accordingly. A patient satisfaction questionnaire was given to the patient to answer at T6.

Data was analyzed statistically using the Statistical Package for the Social Sciences (SPSS), version 20.0. Hair density was expressed as mean \pm standard deviation. Differences between the hair densities at different time points were assessed by one-way repeated measures ANOVA (rmANOVA); post hoc analysis was performed using the Bonferroni test. These tests were two-tailed and $p$ values less than 0.05 considered as statistically significant.

\section{Results}

A total number of 28 subjects were included in the study and after early withdrawal of 4 subjects, who did not complete the treatment protocol, 24 subjects ( 23 males, 1 female) finally completed the study. Patient's characteristics are summarized in Table 1 . The mean age of the participants was 31 years (20-50). According to the Ham- 


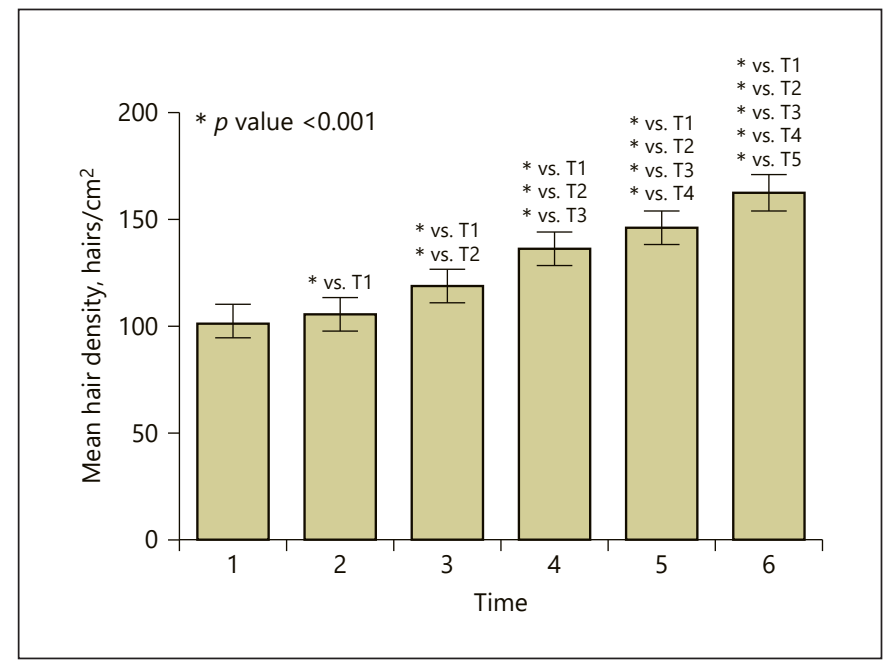

Fig. 1. The effect of AA-PRP injections on hair density. Statistical significance is considered for $p$ values less than 0.05 .

ilton-Norwood staging, 5 males had type II AGA, 9 had type III, 3 had type IV, 3 had type V, 2 had type VI, and 1 had type VII. The female patient had II-1 AGA, according to the Ludwig scale.

Hair density (mean \pm standard deviation) in different evaluation points is presented in Table 2 . Hair density has significantly increased at T2 $(105.46 \pm 18.441)$, at T3 $(118.92 \pm 18.091)$, at T4 $(135.79 \pm 19.060)$, at T5 (145.71 $\pm 19.995)$, and at T6 (161.83 \pm 20.687$)$, when compared with the baseline value (T1) with $p$ values of $<0.001$. The highest hair density was recorded at T6 (Table 2; Fig. 1). The increase rate from baseline value (T1) was 3.14, 16.30, $32.80,42.50$, and $58.27 \%$ at $\mathrm{T} 2, \mathrm{~T} 3, \mathrm{~T} 4, \mathrm{~T} 5$, and T6, respectively.

Macroscopic photographs as in Figures 2 and 3 demonstrate an overall macroscopic view of improvement in hair density and quality. The mean patient satisfaction rate was 72.92 on a linear analogue scale of $0-100(0-$ not satisfied, $100-100 \%$ satisfied with hair growth). At T6, $79.17 \%$ of patients claimed that they like to have a booster session and $87.5 \%$ of them were willing to suggest this as a good treatment modality for another patient with the same disease.

Regarding safety profile of PRP injections, $100 \%$ of patients felt at least mild pain during PRP injections despite local anesthesia. After the procedure, $29.17 \%$ of all patients had a mild pain feeling that lasted up to $2 \mathrm{~h}$. None of the patients had ecchymosis, infection, or keloids as complications.

AA-PRP Injection in Androgenetic Alopecia
Table 1. Patient characteristics

\begin{tabular}{rlll}
\hline No. & Gender & Age, years & Alopecia stage \\
\hline 1 & M & 20 & II \\
2 & M & 35 & II \\
3 & M & 45 & IIIa \\
4 & M & 25 & IIIa \\
5 & M & 30 & VI \\
6 & M & 27 & II \\
7 & M & 50 & VII \\
8 & M & 25 & IVa \\
9 & M & 25 & II \\
10 & M & 28 & II \\
11 & M & 32 & III \\
12 & M & 31 & Va \\
13 & M & 33 & IIIv \\
14 & M & 36 & Va \\
15 & M & 26 & III \\
16 & M & 26 & Va \\
17 & M & 20 & IIIv \\
18 & M & 34 & IV \\
19 & M & 43 & VI \\
20 & F & 39 & II-1 \\
21 & M & 22 & IIIa \\
22 & M & 22 & IIIa \\
23 & M & 45 & IIIa \\
24 & M & 33 & IVa \\
\hline
\end{tabular}

Table 2. Hair density (mean \pm standard deviation) at different time points

\begin{tabular}{ll}
\hline Time point & Hair density, hairs $/ \mathrm{cm}^{2}$ \\
\hline T1 & $102.25 \pm 18.463$ \\
T2 & $105.46 \pm 18.441$ \\
T3 & $118.92 \pm 18.091$ \\
T4 & $135.79 \pm 19.060$ \\
T5 & $145.71 \pm 19.995$ \\
T6 & $161.83 \pm 20.687$ \\
\hline
\end{tabular}

\section{Discussion}

AGA is a common hair loss disorder which does not have much satisfactory therapeutic options. Since characteristic features of AGA include shortening of anagen hair phase causing miniaturization of terminal hair to vellus hair [6], usual treatment modalities of AGA are based on promotion of cellular proliferation and differentiation during hair growth cycle.

Current FDA-approved treatment for AGA is limited to minoxidil and finasteride. Minoxidil acts by prolonging 
Fig. 2. Macrophotographs, 20-year-old male with stage II alopecia. a Before treatment, at T1. b After treatment, at T6 (at 7 months).

Fig. 3. Macrophotographs, 25-year-old male with stage IIIv alopecia. a Before treatment, at T1. b After treatment, at T6 (at 7 months).

Fig. 4. Dermoscopic photographs from a 25-year-old male with stage II alopecia. a Before treatment, at T1. b After treatment, at T6 (at 7 months).
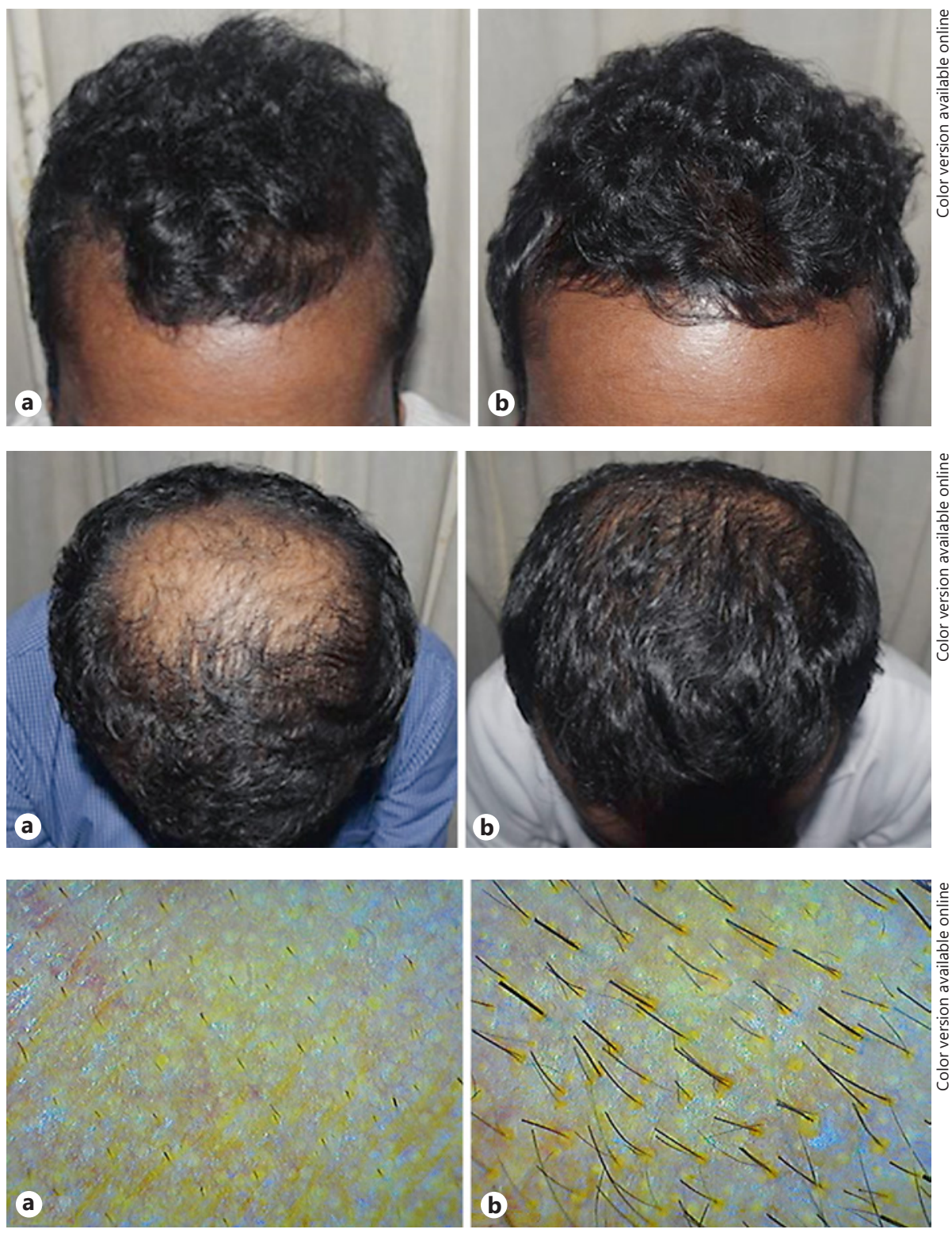

anagen phase and increasing the hair follicle size by stimulating the potassium channels and prostaglandin E2. It also increases dermal papilla cell survival by increasing Bcl-2/ Bax ratio and by activation of extracellular signal-related kinase and Akt $[14,15]$. Finasteride has shown to lengthen the anagen phase leading to subsequent thickened and elongated hairs [16]. It also minimizes the AGA by increased expression of caspases and apoptosis inhibitors and overall activation of hair growth during anagen $[17,18]$.

Anti-apoptotic action of activated PRP is the suggested key contributing factor which stimulates the growth of hair $[19,20]$. Anti-apoptotic regulators, such as the Bcl-2 protein and Akt signaling, are activated by PRP. These prolong the survival of dermal papilla cells during the hair growth cycle, which in turn prolongs anagen phase [10, 19]. Activated PRP was also proven to increase the perifollicular vascular plexus, through the angiogenic ability of increased vascular endothelial growth factor and platelet-derived growth factor levels. This evidence suggests activated PRP as a useful treatment modality for AGA.

In our study, dermoscopic photographs of all 24 patients showed a positive response to treatment with a large variability (29.92\% to $98.51 \%$ ) in increase rate in hair density at T6 when compared with the baseline (T1). Two examples 
Fig. 5. Dermoscopic photographs from a 45-year-old male with stage III alopecia. a Before treatment, at T1. b After treatment, at T6 (at 7 months).
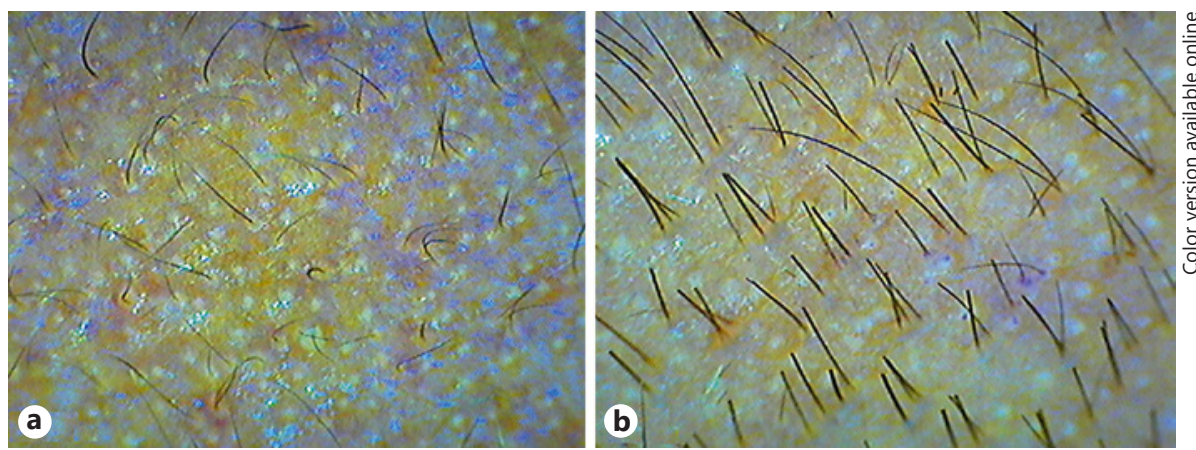

are shown in Figures 4 and 5, with 75.99 and $48.83 \%$ respective increase in hair density from the baseline. Hair density in all the patients followed an upward trend with time. Further, $25 \%$ of patients showed a $>20 \%$ increase in hair density at $\mathrm{T} 3$, and $70.8 \%$ of patients showed a $>50 \%$ increase in hair density at $\mathrm{T} 6$ when compared to $\mathrm{T} 1$.

In this study, the last evaluation (T6) was done after 14 weeks of the last injection of PRP, and in all patients, treatment effect continued at T6. We noticed an increase in hair thickness during analysis of dermoscopic photographs and macroscopic photographs. AA-PRP may influence hair shaft diameter leading to thick hair, but we cannot make a comment, since this study did not assess changes to hair diameter objectively.

One of the advantages of this clinical study is that we used an approved PRP preparation kit (Dr. PRP), since automated kits provide better results as noted in the literature. Further, we detailed the treatment protocol, method of PRP preparation, and administration of PRP, making our results reproducible.

In conclusion, our data suggest that the AA-PRP injections may have a positive therapeutic effect on AGA without major side effects. Further randomized, vehicle-controlled trials are needed to confirm its efficacy compared to potential stimulation by injection alone.

\section{Statement of Ethics}

The study protocol was approved by the ethics review committee - National Hospital of Sri Lanka. Written informed consent was taken from all the patients before participating in the study.

\section{Disclosure Statement}

The authors have no conflicts of interest to declare.

\section{Author Contributions}

Niluka Dilrukshi Paththinige is the principal investigator and was involved in design, conduct of the study, analysis and interpretation of the data, drafting and critical revising of the research work for important intellectual content, and final approval of the version to be published. Janaka K.W. Akarawita is a sub-investigator and was involved in design, conduct of the study, analysis and interpretation of the data, critical revising of the research work for important intellectual content, and final approval of the version to be published. Geetha Jeganathan is a sub-investigator and was involved in design, conduct of the study, analysis and interpretation of the data, conduct of the study, critical revising of the research work for important intellectual content, and final approval of the version to be published.

\section{References}

1 Gan DC, Sinclair RD. Prevalence of male and female pattern hair loss in Maryborough. J Investig Dermatol Symp Proc. 2005 Dec;10(3): 184-9.

2 Alfonso M, Richter-Appelt H, Tosti A, Viera MS, García M. The psychosocial impact of hair loss among men: a multinational European study. Curr Med Res Opin. 2005 Nov; 21(11):1829-36.
3 Cash TF, Price VH, Savin RC. Psychological effects of androgenetic alopecia on women: comparisons with balding men and with female control subjects. J Am Acad Dermatol. 1993 Oct;29(4):568-75.

4 Rathnayake D, Sinclair R. Male androgenetic alopecia. Expert Opin Pharmacother. 2010 Jun;11(8):1295-304.
5 McElwee KJ, Shapiro JS. Promising therapies for treating and/or preventing androgenic alopecia. Skin Therapy Lett. 2012 Jun;17(6):14.

6 Blumeyer A, Tosti A, Messenger A, Reygagne P, Del Marmol V, Spuls PI, et al.; European Dermatology Forum (EDF). Evidence-based (S3) guideline for the treatment of androgenetic alopecia in women and in men. J Dtsch Dermatol Ges. 2011 Oct;9 Suppl 6:S1-57. 
7 Sclafani AP. Applications of platelet-rich fibrin matrix in facial plastic surgery. Facial Plast Surg. 2009 Nov;25(4):270-6.

8 Eppley BL, Woodell JE, Higgins J. Platelet quantification and growth factor analysis from platelet-rich plasma: implications for wound healing. Plast Reconstr Surg. 2004 Nov;114(6):1502-8.

9 Weibrich G, Kleis WK, Hafner G, Hitzler WE. Growth factor levels in platelet-rich plasma and correlations with donor age, sex, and platelet count. J Craniomaxillofac Surg. 2002 Apr;30(2):97-102.

10 Sánchez-González DJ, Méndez-Bolaina E, Trejo-Bahena NI. Platelet-rich plasma peptides: key for regeneration. Int J Pept. 2012; 2012:532519.

11 Tavakkol A, Elder JT, Griffiths CE, Cooper KD, Talwar H, Fisher GJ, et al. Expression of growth hormone receptor, insulin-like growth factor 1 (IGF-1) and IGF-1 receptor mRNA and proteins in human skin. J Invest Dermatol. 1992 Sep;99(3):343-9.
12 Arshdeep, Kumaran MS. Platelet-rich plasma in Dermatology: boon or a bane? Indian J Dermatol. 1992;99:343-9.

13 Uebel CO, da Silva JB, Cantarelli D, Martins $P$. The role of platelet plasma growth factors in male pattern baldness surgery. Plast Reconstr Surg. 2006 Nov;118(6):1458-66.

14 Semalty M, Semalty A, Joshi GP, Rawat MS. Hair growth and rejuvenation: an overview. J Dermatolog Treat. 2011 Jun;22(3):123-32.

15 Han JH, Kwon OS, Chung JH, Cho KH, Eun $\mathrm{HC}$, Kim KH. Effect of minoxidil on proliferation and apoptosis in dermal papilla cells of human hair follicle. J Dermatol Sci. 2004 Apr;34(2):91-8.

16 Tosti A, Piraccini BM. Finasteride and the hair cycle. J Am Acad Dermatol. 2000 May; $42(5$ Pt 1):848-9.
17 Sawaya ME, Blume-Peytavi U, Mullins DL, Nusbaum BP, Whiting D, Nicholson DW, et al. Effects of finasteride on apoptosis and regulation of the human hair cycle. J Cutan Med Surg. 2002 Jan-Feb;6(1):1-9.

18 de Rivero Vaccari JP, Sawaya ME, Brand F 3rd, Nusbaum BP, Bauman AJ, Bramlett HM, et al. Caspase-1 level is higher in the scalp in androgenetic alopecia. Dermatol Surg. 2012 Jul;38(7 Pt 1):1033-9.

19 Li ZJ, Choi HI, Choi DK, Sohn KC, Im M, Seo YJ, et al. Autologous platelet-rich plasma: a potential therapeutic tool for promoting hair growth. Dermatol Surg. 2012 Jul;38(7 Pt 1): 1040-6.

20 Ferraris C, Cooklis M, Polakowska RR, Haake AR. Induction of apoptosis through the PKC pathway in cultured dermal papilla fibroblasts. Exp Cell Res. 1997 Jul;234(1):37-46. 